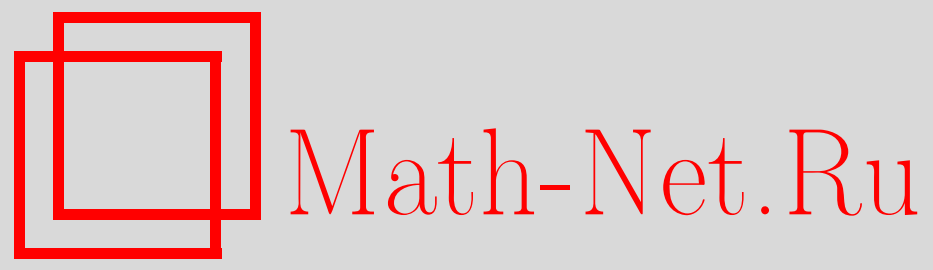

А. А. Каменов, Башелье-версия русского опциона на конечном интервале, Теория вероятн. и ее примен., 2008, том 53, выпуск 3, 576-587

DOI: https://doi.org/10.4213/tvp2451

Использование Общероссийского математического портала Math-Net.Ru подразумевает, что вы прочитали и согласны с пользовательским соглашением

http://www . mathnet.ru/rus/agreement

Параметры загрузки:

IP: 54.198 .187 .58

26 апреля 2023 г., 13:23:44

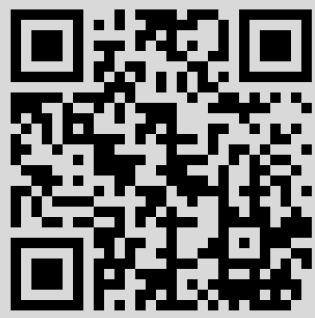




\author{
KAMEHOB A. A.*
}

\title{
БАШЕЛЬЕ-ВЕРСИЯ РУССКОГО ОПЦИОНА НА КОНЕЧНОМ ИНТЕРВАЛЕ
}

\begin{abstract}
Рассматривается задача об оптимальной остановке для русского опциона в модели Башелье в случае конечного временного горизонта. Получено интегральное уравнение, решение которого дает границу между областями остановки и продолжения наблюдений. Найдено асимптотическое поведение данной границы вблизи 0 и на бесконечности.
\end{abstract}

Ключевые слова и фразы: русский опцион, модель Башелье, теория оптимальной остановки, интегральное уравнение, генератор процесса, асимптотическое поведение цены.

1. Термин «русский опцион» был впервые введен Л. Шеппом и А.Н. Ширяевым в работе [1]. В случае бесконечного временного горизонта оптимальное правило остановки формулируется просто: необходимо остановиться, как только разность (в случае модели Башелье) или отношение (в случае модели Блэка-Шоулса) максимума цены актива до настоящего момента и текущей цены достигнет определенного значения.

В том случае если временной горизонт конечен (иными словами, если остановка наблюдений должна произойти не позже некоторого момента времени $T$ ), задача несколько усложняется. Как показано (для случая модели Блэка-Шоулса) в работах [2] и [4], оптимально останавливаться тогда, когда указанное отношение достигает некоторой границы, непрерывно зависящей от времени. Данная работа посвящена исследованию вида этой кривой для модели Башелье.

В п. 2 получено явное интегральное уравнение, решение которого и дает нам искомую кривую. Хотя это уравнение и возможно решить численно, оно достаточно сложно и найти решение в явном виде не представляется возможным. Более того, из этого уравнения не удается даже получить асимптотическое поведение искомой кривой в нуле и на бесконечности. Асимптотика кривой вблизи нуля находится аналогично американскому опциону - рассуждения очень похожи на рассуждения в работе [4] и приводятся здесь скорее для полноты изложения. В случае же асимптотики на бесконечности, хотя используется та же главная

* Московский государственный университет им. М. В. Ломоносова, Ленинские горы, 119992 Москва, Россия; e-mail: kamenov@cnt.ru 
идея, что и в [5], сами рассуждения значительно отличаются от проведенных в этой работе.

2. Предположим, что динамика цен $\left(\widehat{X}_{t}\right)_{t \geqslant 0}$ некоторого финансового актива описывается моделью Башелье:

$$
\widehat{X}_{t}=\mu t+B_{t}
$$

где $B=\left(B_{t}\right)_{t \geqslant 0}$ - стандартное броуновское движение.

Рассматривается задача об оптимальной остановке для платежного поручения

$$
\widehat{V}_{T}=\sup _{\tau \leqslant T} \mathbf{E}\left[\max _{u \leqslant \tau} \widehat{X}_{u}-c \tau\right]
$$

здесь и далее $\tau$ предполагается моментом остановки относительно $\left(\mathscr{F}_{t}^{B}\right)$, где $\mathscr{F}_{t}^{B}=\sigma\left(B_{s}, s \leqslant t\right)$, а $c \geqslant 0$.

Теорема 1. Оптимальным моментом остановки в задаче (2) является момент первого вьхода прочесса $\max _{u \leqslant t} \widehat{X}_{u}-\widehat{X}_{t}$ на кривую $b(t)$, являюшуюся решением интегрального уравнения

$$
\mathbb{A}(t, T, b(t))=b(t)+(c-\mu)(T-t)-c \int_{0}^{T-t} \mathbb{B}(t, u, b(t), b(t+u)) d u
$$

где $\mathbb{A}$ u $\mathbb{B}$ - операторь, явньй вид которьцх указан в (8).

Д ок аз а тель с тв о. Поскольку процесс $\max _{u \leqslant t} \widehat{X}_{u}$ марковским не является и для марковости надо рассматривать процесс $\left(\widehat{X}_{t}, \max _{u \leqslant t} \widehat{X}_{u}\right)$, то поставленная задача двумерна. Тем не менее, как показывает следующая лемма, ее можно свести к одномерной.

Лемма 1. Имеет место представление $\widehat{V}_{T}=\sup _{\tau \leqslant T} \mathbf{E} G\left(X_{\tau}, \tau\right)$, гдe $\operatorname{Law}\left(X_{t} ; t \leqslant T\right)=\operatorname{Law}\left(\max _{u \leqslant t} \widehat{X}_{u}-\widehat{X}_{t} ; t \leqslant T\right) u G(x, t)=x-(c-\mu) t$, $x \geqslant 0$.

Доказ ательство лем мы 1. Воспользуемся тем, что $\max _{u \leqslant t} \widehat{X}_{u}-c t=\left(\max _{u \leqslant t} \widehat{X}_{u}-\widehat{X}_{t}\right)+\left(\widehat{X}_{t}-c t\right)$ и, значит,

$$
\begin{aligned}
\mathbf{E}\left[\max _{u \leqslant \tau} \widehat{X}_{u}-c \tau\right] & =\mathbf{E}\left[\max _{u \leqslant \tau} \widehat{X}_{u}-\widehat{X}_{\tau}\right]+\mathbf{E}\left[\widehat{X}_{\tau}-c \tau\right] \\
& =\mathbf{E}\left(\left[\max _{u \leqslant \tau} \widehat{X}_{u}-\widehat{X}_{\tau}\right]+(\mu-c) \tau\right) .
\end{aligned}
$$

Обозначим $c-\mu=r$ и используем тот факт (доказанный в [2]), что

$$
\operatorname{Law}\left(\max _{u \leqslant t} \widehat{X}_{u}-\widehat{X}_{t} ; t \leqslant T\right)=\operatorname{Law}\left(\left|Y_{t}^{\mu}\right| ; t \leqslant T\right),
$$

где $Y^{\mu}=\left(Y_{t}^{\mu}\right)_{t \leqslant T}$ - это «бэнг-бэнг» процесс, заданный стохастическим дифференциальным уравнением

$$
d Y_{t}^{\mu}=-\mu \operatorname{sign} Y_{t}^{\mu} d t+d B_{t} .
$$


Из соотношений (2)-(4) можно заключить, что достаточно рассматривать новую задачу

$$
V_{T}=\sup _{\tau \leqslant T} \mathbf{E}\left[\left|Y_{\tau}^{\mu}\right|-r \tau\right] .
$$

При этом, очевидно, $V_{T}=\widehat{V}_{T}$.

Процесс $\left|Y^{\mu}\right|$ есть отраженное броуновское движение со сносом: $\left|Y^{\mu}\right|=\operatorname{RBM}(-\mu)=X$, где процесс $X$ определяется инфинитезимальным генератором $\mathbb{L}_{X}=-\mu f^{\prime}+\frac{1}{2} f^{\prime \prime}$ с $f^{\prime}(0+)=0$. Таким образом, (5) можно записать как $V_{T}=\sup _{\tau \leqslant T} \mathbf{E} G\left(X_{\tau}, \tau\right)$, где $G(x, t)=x-r t, x \geqslant 0$. Лемма 1 доказана.

Итак, исходная задача сведена к одномерной. Теперь рассмотрим последовательность задач

$$
V(t, x)=\sup _{0 \leqslant \tau \leqslant T-t} \mathbf{E}_{t, x} G\left(X_{t+\tau}, t+\tau\right),
$$

где $\mathbf{E}_{t, x}$ обозначает условное математическое ожидание $\mathbf{E}\left(\cdot \mid X_{t}=x\right)$.

По формуле Ито $d X_{t}=d\left|Y_{t}^{\mu}\right|=-\mu d t+\operatorname{sign} Y_{t}^{\mu} d B_{t}+d L^{0}\left(Y^{\mu}\right)_{t}$ (здесь $L^{0}$ обозначает локальное время Леви процесса в нуле) и, следовательно, $V\left(t+s, X_{t+s}\right)$ равно

$$
\begin{array}{r}
V\left(t, X_{t}\right)+\int_{0}^{s}\left[\left(V_{t}^{\prime}-\mu V_{x}^{\prime}+\frac{1}{2} V_{x x}^{\prime \prime}\right)\left(t+u, X_{t+u}\right) d u\right. \\
\left.+V_{x}^{\prime}\left(t+u, X_{t+u}\right) d L^{0}\left(Y^{\mu}\right)_{u}\right]+M_{s}
\end{array}
$$

где $M_{s}=\int_{0}^{s} V_{x}^{\prime}\left(t+u, X_{t+s}\right) \operatorname{sign} Y_{u}^{\mu} d B_{t}$.

Из принципа нормального отражения следует, что при $x=0$, т.е. в точках роста $L^{0}\left(Y^{\mu}\right)_{u}$ выполнено равенство $V_{x}(t, x)=0$, значит, $\int_{0}^{s} V_{x}(t+$ $\left.u, X_{t+u}\right) d L^{0}\left(Y^{\mu}\right)_{u}=0$.

Можно надеяться, что оптимальный момент остановки в задачах (6) определяется некоторой границей $b=(b(t))_{t \leqslant T}$, разделяющей области продолжения наблюдений $C_{T}=\{(t, x), t \leqslant T, x \geqslant 0: x<b(t)\}$ и остановки наблюдений $D_{T}=\{(t, x), t \leqslant T, x \geqslant 0: x \geqslant b(t)\}$. По-видимому, кривая $b=b(t)$ имеет форму, указанную на рис. 1.

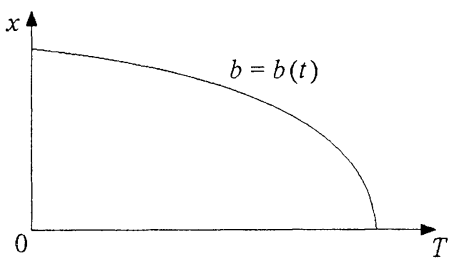

Puc. 1 
Предположим, что области остановки и продолжения наблюдений имеют указанный вид для некоторой убывающей функции $b(t)$. Тогда выражение (7) можно записать следуюшим образом:

$$
\begin{aligned}
V\left(t, X_{t}\right)+\int_{0}^{s} & \left(\mathbf{I}\left(X_{t+u} \geqslant b(t+u)\right)\left[V_{t}^{\prime}-\mu V_{x}^{\prime}+\frac{1}{2} V_{x x}^{\prime \prime}\right]\left(t+u, X_{t+u}\right)\right. \\
& +\mathbf{I}\left(X_{t+u}<b(t+u)\right) \\
& \left.\times\left[V_{t}^{\prime}-\mu V_{x}^{\prime}+\frac{1}{2} V_{x x}^{\prime \prime}\right]\left(t+u, X_{t+u}\right)\right) d u+M_{s}
\end{aligned}
$$

При этом там, где наблюдения продолжаются, т.е. там, где выполнено неравенство $X_{t+u}<b(t+u)$, имеет место $V_{t}^{\prime}-\mu V_{x}^{\prime}+\frac{1}{2} V_{x x}^{\prime \prime}=0$. Действительно, двумерный процесс $Z_{t}=\left(X_{t}, t\right)$ - марковский, его генератор $\mathbb{L}_{Z}$ равен $\frac{\partial}{\partial t}+\mathbb{L}_{X}=\frac{\partial}{\partial t}-\mu \frac{\partial}{\partial x}+\frac{1}{2} \frac{\partial^{2}}{\partial x^{2}}$. Как показано в [3, гл. 3 , п. 2.1], в области продолжения наблюдений имеет место равенство $\mathbb{L}_{Z} V=0$, т.е., с учетом вида $\mathbb{L}_{Z}$, имеем $V_{t}^{\prime}-\mu V_{x}^{\prime}+\frac{1}{2} V_{x x}^{\prime \prime}=0$.

Там же, где наблюдения останавливаются, выполнены равенства $V_{t}^{\prime}-\mu V_{x}^{\prime}+\frac{1}{2} V_{x x}^{\prime \prime}=G_{t}^{\prime}-\mu G_{x}^{\prime}+\frac{1}{2} G_{x x}^{\prime \prime}=-c$. Следовательно,

$$
V\left(t+s, X_{t+s}\right)=V\left(t, X_{t}\right)-c \int_{0}^{s} \mathbf{I}\left(X_{t+u} \geqslant b(t+u)\right) d u+M_{s} .
$$

Положим $s=T-t, X_{t}=x$ :

$$
V\left(T, X_{T}\right)=V(t, x)-c \int_{0}^{T-t} \mathbf{I}\left(X_{t+u} \geqslant b(t+u)\right) d u+M_{s} .
$$

Беря математическое ожидание в левой и правой части, находим

$$
\mathbf{E}_{t, x} V\left(T, X_{T}\right)=V(t, x)-c \int_{0}^{T-t} \mathbf{E}_{t, x}\left[\mathbf{I}\left(X_{t+u} \geqslant b(t+u)\right)\right] d u .
$$

Если $x=b(t)$, то $\mathbf{E}_{t, b(t)}\left[X_{T}-r T\right]=V(t, b(t))-c \int_{0}^{T-t} \mathbf{E}_{t, b(t)}\left[\mathbf{I}\left(X_{t+u} \geqslant\right.\right.$ $b(t+u))] d u$, или

$$
\mathbf{E}_{t, b(t)} X_{T}=b(t)+r(T-t)-c \int_{0}^{T-t} \mathbf{E}_{t, b(t)}\left[\mathbf{I}\left(X_{t+u} \geqslant b(t+u)\right)\right] d u .
$$

Формально, если положить

$$
\mathbb{A}(t, T, b(t))=\mathbf{E}_{t, b(t)} X_{T}, \quad \mathbb{B}(t, u, b(t), b(t+u))=\mathbf{E}_{t, b(t)} \mathbf{I}\left(X_{t+u} \geqslant b(t+u)\right),
$$

то для нахождения $b$ необходимо решить интегральное уравнение

$$
\mathbb{A}(t, T, b(t))=b(t)+(c-\mu)(T-t)-c \int_{0}^{T-t} \mathbb{B}(t, u, b(t), b(t+u)) d u .
$$


Итак, с учетом сделанного предположения о виде оптимального правила остановки, мы доказали теорему 1. Доказательство того, что это предположение верно, приведено в следующем пункте. Сейчас рассмотрим полученное уравнение подробнее для частного случая.

Пусть $\mu=0$, т.е. мы рассматриваем задачу

$$
\widehat{V}_{T}=\sup _{\tau \leqslant T} \mathbf{E}\left[\max _{u \leqslant \tau} B_{u}-c \tau\right]=\sup _{\tau \leqslant T} \mathbf{E}\left[\left|B_{\tau}\right|-c \tau\right] .
$$

Тогда $\mathbb{A}(t, T, b(t))$ будет равно $\mathbf{E}\left|B_{T-t}+b(t)\right|=\mathbf{E}\left|\sqrt{T-t} B_{1}+b(t)\right|$, а $\mathbb{B}(t, u, b(t), b(t+u))=\mathbf{E} \mathbf{I}\left(\left|B_{u}+b(t)\right|>b(t+u)\right)=\mathbf{P}\left\{\left|\sqrt{u} B_{1}+b(t)\right|>\right.$ $b(t+u)\}$. Таким образом, интегральное уравнение (9) принимает вид:

$\mathbf{E}\left|\sqrt{T-t} B_{1}+b(t)\right|=b(t)+c(T-t)-c \int_{0}^{T-t} \mathbf{P}\left\{\left|\sqrt{u} B_{1}+b(t)\right|>b(t+u)\right\} d u$.

Далее,

$$
\begin{aligned}
& \mathbf{E}\left|\sqrt{T-t} B_{1}+b(t)\right|=\sqrt{T-t} \mathbf{E}\left|B_{1}+\frac{b(t)}{\sqrt{T-t}}\right| \\
& \quad=\sqrt{T-t}\left[\frac{b(t)}{\sqrt{T-t}}\left(1-2 \Phi\left(-\frac{b(t)}{\sqrt{T-t}}\right)\right)+\sqrt{\frac{2}{\pi}} \exp \left\{-\frac{b^{2}(t)}{2(T-t)}\right\}\right]
\end{aligned}
$$

и

$$
\begin{aligned}
\mathbf{P}\left\{\left|\sqrt{u} B_{1}+b(t)\right|>b(t+u)\right\}= & 1-\Phi\left(\frac{b(t+u)-b(t)}{\sqrt{u}}\right) \\
& +\Phi\left(\frac{-b(t+u)-b(t)}{\sqrt{u}}\right) .
\end{aligned}
$$

Подставляя эти выражения в (10), получаем

$$
\begin{aligned}
& \sqrt{\frac{2}{\pi}(T-t)} \exp \left\{-\frac{b^{2}(t)}{2(T-t)}\right\}-2 b(t) \Phi\left(-\frac{b(t)}{\sqrt{T-t}}\right) \\
& =c \int_{0}^{T-t}\left[\Phi\left(\frac{b(t+u)-b(t)}{\sqrt{u}}\right)-\Phi\left(\frac{-b(t+u)-b(t)}{\sqrt{u}}\right)\right] d u .
\end{aligned}
$$

Для того чтобы численно решить это уравнение, преобразуем его. Введем переменную $s=T-t$ и обозначим $h(s)=b(T-s)$, тогда уравнение примет вид

$$
\begin{aligned}
& \sqrt{\frac{2 s}{\pi}} \exp \left\{-\frac{h^{2}(s)}{2 s}\right\}-2 h(s) \Phi\left(-\frac{h(s)}{\sqrt{s}}\right) \\
& =c \int_{0}^{s}\left[\Phi\left(\frac{h(s-u)-h(s)}{\sqrt{u}}\right)-\Phi\left(\frac{-h(s-u)-h(s)}{\sqrt{u}}\right)\right] d u .
\end{aligned}
$$


В таком виде уравнение допускает приближенное решение (см. рис. 2), поскольку замена слагаемого $2 h(s) \Phi(-h(s) / \sqrt{s})$ на $2 h(s+$ $\alpha) \Phi(-h(s+\alpha) / \sqrt{s+\alpha})$ приводит к тому, что значение в точке $s+\alpha$ восстанавливается по значениям на отрезке $[0, s]$.

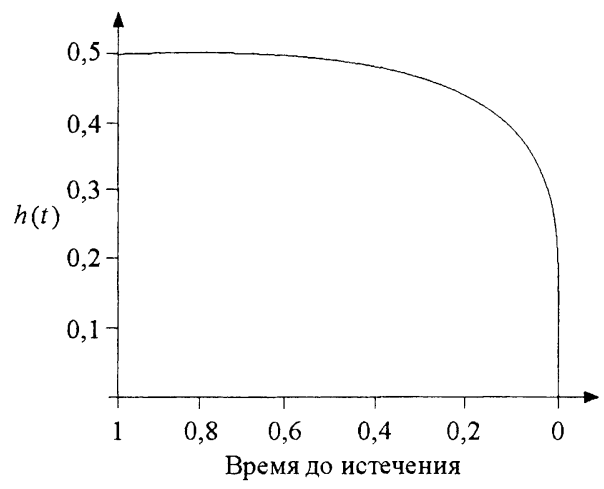

Рис. 2. Результат численного моделирования границы $h(t)$ (приближение шагов, случай $c=1$ )

Заметим, что при $T=\infty$ решение этой задачи просто: используя тождество $\mathbf{E} \tau=\mathbf{E} B_{\tau}^{2}$, получаем $V_{\infty}=\sup _{\tau<\infty} \mathbf{E}\left[\left|B_{\tau}\right|-c B_{\tau}^{2}\right]$. Заметив, что функция $|x|-c x^{2}$ достигает максимума в точках $|x|=1 /(2 c)$, получаем, что оптимальный момент остановки $\tau^{*}$ в этом случае равен $\inf \left\{t:\left|B_{t}\right|=1 /(2 c)\right\}$ и $V_{\infty}=1 /(2 c)-c(1 /(2 c))^{2}=1 /(4 c)$. Это позволяет проверять результаты численного моделирования, поскольку, как будет показано далее, $h(t)$ при $t \rightarrow \infty$ сходится к $1 /(2 c)$.

3. Теперь докажем, что момент выхода процесса $X_{t}$ на границу $b(t)$ действительно оптимален в исходной задаче об оптимальной остановке. Идейно доказательство повторяет приведенное в книге [3] для случая геометрического броуновского движения, однако некоторые существенные отличия все же имеются.

Для установления некоторых свойств функции $V(t, x)$ нам понадобится ее представление через $\widehat{X}_{t}$. Заметим, что $\mathbf{E}_{t, x}$ есть условное математическое ожидание при условии события $\left\{\widehat{M}_{t}-\widehat{X}_{t}=x\right\}$, где мы обозначили $\widehat{M}_{t}=\max _{0 \leqslant s \leqslant t} \widehat{X}_{s}$. Итак, $V(t, x)=\sup _{0 \leqslant \tau \leqslant T-t} \mathbf{E}\left(\widehat{M}_{t+\tau}-\right.$ $\left.\widehat{X}_{t+\tau}-c(t+\tau) \mid \widehat{M}_{t}-\widehat{X}_{t}=x\right)$. Заметим, что данное условное математическое ожидание не зависит от $\widehat{X}_{t}$, следовательно, его можно записать в виде $\mathbf{E}\left(\widehat{M}_{t+\tau}-\widehat{X}_{t+\tau}-c(t+\tau) \mid \widehat{M}_{t}=x, \widehat{X}_{t}=0\right)$. Вводя процесс $\widetilde{X}_{t}$, также являющийся броуновским движением со сносом $\mu$ и с условием $\widetilde{X}_{0}=0$, получаем, что

$$
\begin{aligned}
V(t, x) & =-c t+\sup _{0 \leqslant \tau \leqslant T-t} \mathbf{E}\left(\max \left(\widetilde{M}_{\tau}-\widetilde{X}_{\tau}, x\right)-c \tau\right) \\
& =-c t+\sup _{0 \leqslant \tau \leqslant T-t} \mathbf{E}\left(\max \left(X_{\tau}, x\right)-c \tau\right),
\end{aligned}
$$


где $\widetilde{M}_{t}=\max _{0 \leqslant s \leqslant t} \tilde{X}_{s}$. Заметим теперь, что функция под знаком математического ожидания возрастает и выпукла вниз по $x$ при любом $\tau$, значит, этими же свойствами обладает и $V(t, x)$.

Лемма 2. Функция $V: S=[0, T] \times[0, \infty) \rightarrow \mathbb{R}$ является непрерьвноน̆.

Д о к а з а т е л с с в о. Воспользовавшись представлением (11) математического ожидания, получим для $x>y$ :

$$
\begin{aligned}
& V(t, x)-V(t, y) \\
& \quad=\sup _{0 \leqslant \tau \leqslant T-t} \mathbf{E}\left(\max \left(X_{\tau}, x\right)-c \tau\right)-\sup _{0 \leqslant \tau \leqslant T-t} \mathbf{E}\left(\max \left(X_{\tau}, y\right)-c \tau\right) \\
& \quad \leqslant \sup _{0 \leqslant \tau \leqslant T-t}\left(\max \left(X_{\tau}, x\right)-\max \left(X_{\tau}, y\right)\right) \leqslant x-y .
\end{aligned}
$$

Таким образом, доказана равномерная непрерывность по $x$, и для доказательства непрерывности по паре переменных осталось доказать непрерывность по $t$ при каждом фиксированном $x$. Действительно, пусть $0 \leqslant t_{1}<t_{2} \leqslant T$ и задано некоторое $\varepsilon>0$. Тогда возьмем момент остановки $\tau_{1}^{\varepsilon}$ таким, чтобы выполнялось неравенство $\mathbf{E}_{t_{1}, x} G\left(X_{t_{1}+\tau_{1}^{\varepsilon}}, t_{1}+\tau_{1}^{\varepsilon}\right) \geqslant$ $V\left(t_{1}, x\right)-\varepsilon$. Положим $\tau_{2}^{\varepsilon}=\min \left(\tau_{1}^{\varepsilon}, T-t_{2}\right)$. Пользуясь очевидным неравенством $V\left(t_{2}, x\right) \geqslant \mathbf{E}_{t_{2}, x} G\left(X_{t_{2}+\tau_{2}^{\varepsilon}}, t_{2}+\tau_{2}^{\varepsilon}\right)$ и обозначая условные распределения $X$ при условии $X_{t_{1}}=x$ через $X^{1}$, а при условии $X_{t_{2}}=x$ через $X^{2}$, получим

$$
0 \leqslant V\left(t_{1}, x\right)-V\left(t_{2}, x\right) \leqslant \mathbf{E}\left(X_{t_{1}+\tau_{1}^{\varepsilon}}^{1}-X_{t_{2}+\tau_{2}^{\varepsilon}}^{2}-r\left(\tau_{1}^{\varepsilon}-\tau_{2}^{\varepsilon}\right)\right)+\varepsilon .
$$

Далее, устремим сначала $t_{2}-t_{1}$ к 0 (при этом $\tau_{1}^{\varepsilon}-\tau_{2}^{\varepsilon} \rightarrow 0$ и математическое ожидание справа также устремится к 0 ), а потом $\varepsilon$ к 0 . Тогда $V\left(t_{1}, x\right)-V\left(t_{2}, x\right) \rightarrow 0$, что и требовалось для доказательства непрерывности $V$.

Введем множество продолжения наблюдений $C=\{(t, x) \in[0, T] \times$ $[0, \infty): V(t, x)>G(x)\}$ и множество остановки $D=\{(t, x) \in[0, T] \times$ $[0, \infty): V(t, x)=G(x)\}$. Поскольку функции $V$ и $G$ непрерывны, множество $C$ открыто, а $D$, соответственно, замкнуто. С помощью стандартных рассуждений, основанных на строго марковском свойстве, получим, что момент остановки $\tau_{D}=\inf \left\{0 \leqslant s \leqslant T-t:\left(t+s, X_{t+s}\right) \in D\right\}$ является оптимальным в задаче (6).

Покажем, что множество продолжения наблюдений $C$ действительно задается как $C=\{(t, x): x<b(t)\}$ для некоторой убывающей функции $b(t)$.

В самом деле, предположим, что $(t, x) \in C$. Тогда, воспользовавшись неравенством (12), получим для $y<x$ :

$$
V(t, y)-y \geqslant V(t, x)-x>0
$$


А значит, $(t, y)$ также принадлежит $C$. Таким образом, области остановки и продолжения наблюдений разделяются графиком некоторой функции $b(t)$. То, что $b(t)$ убывающая, сразу следует из доказанного ранее убывания $V(t, x)$ по $t$.

Для окончательного решения поставленной задачи осталось показать, что $b(t)$ является единственным решением интегрального уравнения (9). Соответствующее доказательство в нашем случае практически не отличается от доказательства, проведенного в $[3$, гл. 7, п. 2.2], поэтому приводить мы его здесь не будем.

4. Заметим, что если мы позволим менять $T$, то $b(t, T)$ будет зависеть только от $T-t$, поэтому вместо исследования вида кривой $b$ мы будем работать с уже введенной функцией $h(t)=b(T-t)$. Очевидно, что $h(0)=0$ и $h(t)$ возрастает по $t$. Обозначим $U(t, x)=V_{t}(0, x)=$ $c(T-t)+V_{T}(T-t, x)$.

Исследуем сначала асимптотику $b(t)$ при $t \rightarrow T$.

Теорема 2. $\Pi p u t \rightarrow T$

$$
b(t)=\sqrt{(T-t) \ln (T-t)^{-1}}+O(T-t) .
$$

Д ок аз а т е л с т в о. Сразу перейдем от рассмотрения асимптотики $b(t)$ при $t \rightarrow T$ к изучению асимптотики $h(t)$ при $t \rightarrow 0$. Тогда утверждение теоремы можно сформулировать так:

$$
h(t)=\sqrt{t \ln t^{-1}}+O(t) .
$$

Воспользовавшись тем, что $\operatorname{Law}\left(X_{t}\right)=\operatorname{Law}\left(\max _{u \leqslant t} \widehat{X}_{u}-\widehat{X}_{t}\right)$, напишем:

$$
\mathbf{E} X_{t}=\mathbf{E}\left(\max _{u \leqslant t} \widehat{X}_{u}-\widehat{X}_{t}\right)=\mathbf{E} \max _{u \leqslant t}\left(\mu u+B_{u}\right)-\mu t .
$$

Далее, очевидно, что $\max _{u \leqslant t}\left(\mu u+B_{u}\right) \geqslant \max _{u \leqslant t} B_{u}+\min _{u \leqslant t} \mu u$, при этом второе слагаемое равно $\min (\mu t, 0)$. Математическое ожидание максимума броуновского движения до момента $t$, как известно, равно $\sqrt{t \pi} / 2$. Итак, получено неравенство

$$
\mathbf{E} X_{t} \geqslant \sqrt{\frac{t \pi}{2}}-\mu t+\min (\mu t, 0)=\sqrt{\frac{t \pi}{2}}-\mu^{+} t
$$

где $\mu^{+}=\max (\mu, 0)$.

Рассмотрим функцию $W(x)=\mathbf{E}_{x} X_{t}-r t$, где $\mathbf{E}_{x}$ обозначает условное математическое ожидание при условии $X_{0}=x$. Заметим, что если $t$ мало, то $W(x) \geqslant W(0)>0$ в силу найденной асимптотики для $\mathbf{E} X_{t}$, а значит, при достаточно малых $x$ выполнено неравенство $W(x)>x$. При $x>h(t)$ имеем $W(x) \leqslant U(t, x)=x$. В силу выпуклости вниз $W(x)$ как функции от $x$, уравнение $W(x)=x$ при фиксированном $t$ имеет единственный положительный корень $a(t)$. 
Лемма 3. Если $t \rightarrow 0$, mo $a(t)-h(t)=O(\sqrt{t})$.

Д ок а з а т е ль с т в о. Заметим, что $a(t)<h(t)$, поскольку $a(t)=W(a(t))<U(t, a(t))$, а значит, $a(t)$ лежит в области продолжения наблюдений. Далее, поскольку $U(t, x) \in C^{2}$ по $x$, то $U(t, a(t))$ может быть представлено в виде

$$
U(t, a(t))=U(t, h(t))+(a(t)-h(t)) U_{x}^{\prime}(t, h(t))+\frac{1}{2}(a(t)-h(t))^{2} U_{x x}^{\prime \prime}(t, \xi),
$$

где $a(t)<\xi<h(t)$. Условие гладкого склеивания для $V(t, x)$ дает равенство $U_{x}^{\prime}(t, h(t))=1$, а $U(t, h(t))+a(t)-h(t)=a(t)=W(a(t))$. Таким образом, имеем

$$
U(t, a(t))-W(a(t))=\frac{1}{2}(a(t)-h(t))^{2} U_{x x}^{\prime \prime}(t, \xi) .
$$

C другой стороны, заметим, что $U(t, x)=\sup _{0 \leqslant \tau \leqslant t} \mathbf{E}\left(\max _{u \leqslant \tau} \widehat{X}_{u} \vee\right.$ $x-c \tau) \leqslant \mathbf{E}\left(\max _{u \leqslant t} \hat{X}_{u} \vee x\right)=\mathbf{E}_{x} X_{t}+\mu t$ и

$$
U(t, x)-W(x) \leqslant \mathbf{E}_{x} X_{t}+\mu t-\mathbf{E}_{x} X_{t}+r t=c t .
$$

Также имеем $\frac{1}{2} U_{x x}^{\prime \prime}=\mu U_{x}^{\prime}+U_{t}$. Далее, $U_{t}^{\prime} \geqslant 0$ в силу возрастания $U(t, x)$ по $t$. Поскольку также $U_{x}^{\prime} \geqslant 0$, то и $U_{x x}^{\prime \prime} \geqslant 0$. Это означает, что $U_{x}^{\prime}(t, \xi) \geqslant U(t, \xi) / \xi \geqslant 1$. С учетом вышесказанного,

$$
(a(t)-h(t))^{2}=2 \frac{U(t, a(t))-W(a(t))}{U_{x x}^{\prime \prime}(t, \xi)} \leqslant \frac{2 c t}{\mu U_{x}^{\prime}+U_{t}^{\prime}} \leqslant \frac{2 c t}{\mu} .
$$

Лемма 3 доказана; значит, для того чтобы найти асимптотику $h(t)$, достаточно исследовать поведение $a(t)$ вблизи 0 .

Лемма 4. При $t \rightarrow 0$ верно $a(t) \sim \sqrt{t \ln t^{-1}}$.

Д ок аз а т е льств о. По определению $a(t)+r t=\mathbf{E}_{a(t)} X_{t}=$ $\mathbf{E}\left(\max _{u \leqslant t} \widehat{X}_{u} \vee a(t)\right)-\mu t$, или $\mathbf{E}\left(\max _{u \leqslant t} \widehat{X}_{u}-a(t)\right)^{+}=(r+\mu) t=c t$. Но $\max _{u \leqslant t} \widehat{X}_{u}-\mu^{+} t \leqslant \max _{u \leqslant t} B_{u} \leqslant \max _{u \leqslant t} \widehat{X}_{u}+\mu^{-} t$. Отсюда следует, что $0<c-\mu^{-} \leqslant t^{-1} \mathbf{E}\left(\max _{u \leqslant t} \widehat{B}_{u}-a(t)\right)^{+} \leqslant c+\mu^{+}$. Далее, $\mathbf{E}\left(\max _{u \leqslant t} B_{u}-a(t)\right)^{+}=\sqrt{t} \mathbf{E}\left(\max _{u \leqslant 1} B_{u}-\alpha(t)\right)^{+}$, где $\alpha(t)=a(t) / \sqrt{t}$. Таким образом, $\mathbf{E}\left(\max _{u \leqslant 1} B_{u}-\alpha(t)\right)^{+}=O(\sqrt{t})$, что возможно лишь в том случае, когда $\alpha(t) \rightarrow \infty$ при $t \rightarrow 0$. Далее, обозначая $\max _{u \leqslant 1} B_{u}=\xi$, получаем

$$
\begin{aligned}
\mathbf{E}(\xi-\alpha)^{+} & =\sqrt{\frac{2}{\pi}} \int_{\alpha}^{\infty}(x-\alpha) e^{-x^{2} / 2} d x \\
& =\sqrt{\frac{2}{\pi}} \frac{e^{-\alpha^{2} / 2}}{\alpha^{2}} \int_{0}^{\infty} y e^{-y-y^{2} /\left(2 \alpha^{2}\right)} d y \sim \sqrt{\frac{2}{\pi}} \frac{e^{-\alpha^{2} / 2}}{\alpha^{2}}
\end{aligned}
$$


при $t \rightarrow 0$. Следовательно, $0<m<\alpha^{2} e^{\alpha^{2} / 2} \sqrt{t}<M$, откуда $-\ln t+\ln m<$ $\alpha^{2}<-\ln t+\ln M$, что означает эквивалентность $\alpha(t) \sim \ln t^{-1}$ при $t \rightarrow 0$, т.е. дает необходимую асимптотику для $a(t)$.

Итак, найдя асимптотику $a(t)$ при $t \rightarrow 0$, мы доказали утверждение (15), а значит, и теорему 2.

5. Исследуем асимптотику $h(t)$ на бесконечности. В случае бесконечного временного горизонта оптимальным моментом остановки является момент первого достижения процессом $X_{t}$ некоторого значения $S_{0}$, и было бы логично ожидать, что $h(t) \rightarrow S_{0}$ при $t \rightarrow \infty$. Для того чтобы доказать это и найти, насколько сходимость быстрая, нам понадобится оценить разность между ценами опционов в нулевой момент времени для случаев конечного и бесконечного горизонтов.

Итак, сначала решим исходную задачу в случае бесконечного временного горизонта. Имеем следующую систему:

$$
\begin{array}{lllll}
\frac{1}{2} V_{\infty}^{\prime \prime}(x)-\mu V_{\infty}^{\prime}(x)=c \quad \text { при } & x \leqslant S_{0}, & & \\
V_{\infty}^{\prime}(x)=0 & \text { при } \quad x=0, & V_{\infty}^{\prime}(x)=1 & \text { при } & x=S_{0}, \\
V_{\infty}(x)=x & \text { при } \quad x \geqslant S_{0}, & V_{\infty}(x)>x & \text { при } & x<S_{0} .
\end{array}
$$

Решив эту систему, получаем, что $V_{\infty}(x)=a_{1} e^{2 \mu x}+a_{2}-c x / \mu$ на отрезке $\left[0, S_{0}\right]$, где

$$
S_{0}=\frac{\ln (\mu / c+1)}{2 \mu}, \quad a_{1}=\frac{c}{2 \mu^{2}}, \quad a_{2}=\frac{(1+c / \mu)(\ln (\mu / c+1)-1)}{2 \mu} .
$$

Заметим, что в тех точках, где $\mathbb{L}_{X} V=V_{t}^{\prime}-\mu V_{x}^{\prime}+\frac{1}{2} V_{x x}^{\prime \prime}=0$, выполнено равенство $\mathbb{L}_{1} U=c$, где $\mathbb{L}_{1}=-\partial / \partial t-\mu \partial / \partial x+\frac{1}{2} \partial^{2} / \partial x^{2}$ ( $\mathbb{L}_{1}$ отличается от $\mathbb{L}_{X}$ знаком при $\left.\partial / \partial t\right)$.

Теорема 3. Для некоторого положительного $D$ имеет место оченка $0<V_{\infty}(S)-U(t, S)<D e^{x \mu / 2-q t} \cos (\lambda x)$, где $\lambda=\pi /\left(2 S_{0}\right) u$ $q=(\min (\mu, 0))^{2} / 4+\lambda^{2}$.

Д ок азат ель с т в о. Зафиксируем некоторое число $\xi>0$ и обозначим $D_{\xi}=\{(x, t): 0 \leqslant x \leqslant 1 /(2 c), 0 \leqslant t \leqslant \xi\}$. В этой области $V_{\infty}(S)>U(t, S)$.

Оценим разность между $V_{\infty}(S)$ и $U(t, S)$ величиной $H(S, t)$ вида $H_{1}(S) H_{2}(t)$, удовлетворяющей условию $\mathbb{L}_{1} H \leqslant 0$. В качестве такой величины можем взять $D e^{p S+q t} \cos (\lambda S)$, где $\lambda=\pi /\left(2 S_{0}\right)$. Заметим, что это выражение неотрицательно при $0 \leqslant S \leqslant S_{0}$. Указанное условие дает нам два неравенства: $p \leqslant \mu / 2$ и $q-\mu p+p^{2}-\lambda^{2} \leqslant 0$. Нам хотелось бы, чтобы $q$ было возможно больше (чтобы получить максимально сильную оценку), но при этом, как будет видно позднее, $p$ должно быть неположительным. В таком случае максимальное $q$ можем взять при $p=\min (\mu, 0) / 2$ : тогда $q=-(\min (\mu, 0))^{2} / 4-\lambda^{2}$. 
Введем величины $w=V_{\infty}-U-H$ и $\widetilde{w}=V_{\infty}-H$.

Лемма 5. Максимум функиии $w$ в области $D_{\xi}$ достигается на гранище этой области.

Док аз а те ль с т в о ле м мы 5 . При $S<h(t)$ имеем $\mathbb{L}_{1} w=$ $\mathbb{L}_{1} V_{\infty}-\mathbb{L}_{1} U-\mathbb{L}_{1} H=c-c-\mathbb{L}_{1} H \geqslant 0$. При $S>h(t)$ также $\mathbb{L}_{1} w=$ $\mathbb{L}_{1} V_{\infty}-\mathbb{L}_{1} U-\mathbb{L}_{1} H=c-\mu-\mathbb{L}_{1} H \geqslant r \geqslant 0$.

Во внутренних точках $D_{\xi}$, для которых $S \neq h(t), w$ не может достигать максимума, иначе мы получили бы, что $\mathbb{L}_{1} w<0$, что не так.

Предположим, что максимум достигается в точке $\left(h\left(t_{0}\right), t_{0}\right)$. Тогда в этой точке $\widetilde{w}_{S}^{\prime}=U_{S}^{\prime}=1$. Также, очевидно, $\widetilde{w}_{t}^{\prime \prime}\left(h\left(t_{0}\right), t_{0}\right) \geqslant 0$ и $\widetilde{w}_{S S}^{\prime \prime}\left(h\left(t_{0}\right), t_{0}\right) \leqslant 0$. Учитывая все это, получаем: $\mathbb{L}_{1} \widetilde{w}\left(h\left(t_{0}\right), t_{0}\right) \leqslant-\mu$. Но, с другой стороны, $\mathbb{L}_{1} \widetilde{w}\left(h\left(t_{0}\right), t_{0}\right) \geqslant c$, что делает достижение величиной $w$ максимума на кривой $h(t)$ невозможным.

Таким образом, $w$ должна достигать своего максимума на границе $D_{\xi}$. При $S=0$ она не может его достичь, поскольку $w_{x}^{\prime}(0, t)=$ $-H_{x}^{\prime}(0, t)=-p H(0, t) \geqslant 0$, при этом в случае равенства нулю имеем $w_{x}^{\prime \prime}(0, t)=-H_{x}^{\prime \prime}(0, t)=\lambda^{2} H(0, t) \geqslant 0$. При $S=S_{0}$ имеем $V_{\infty}=U=S_{0}$, $w=-H<0$.

Лемма 6. Существует такое $D$, ито при $t=0 u x \in\left[0, S_{0}\right]$ имеет место неравенство $w \leqslant 0$.

Док а за тель с тво ле м мы 6 . Заметим, что $V_{\infty}(S)-$ $U(0, S)=e^{p S} \cos (\lambda S)$, что равно нулю при $S=S_{0}$, причем производная первой функции равна нулю, а производная второй есть $p e^{p S_{0}} \cos \left(\lambda S_{0}\right)-$ $\lambda e^{p S_{0}} \sin \left(\lambda S_{0}\right)=-\lambda e^{p S_{0}}<0$. Это означает, что, будучи доопределенной нулем в точке $S_{0}$, функция $(V-U) /\left(e^{p S} \cos (\lambda S)\right)$ непрерывна на отрезке $\left[0, S_{0}\right]$ и достигает на нем максимума. Это максимальное значение и можно взять в качестве $D$.

Таким образом, если максимум функции $w$ в области $D_{\xi}$ положителен, то он достигается на отрезке $\left\{(x, \xi): x \in\left[0, S_{0}\right]\right\}$. При $\xi \rightarrow \infty$ максимум не будет уменьшаться и при всех $\xi$ будет достигаться на указанном отрезке. Но, с другой стороны, $\lim _{\xi \rightarrow \infty}\left(V_{\infty}-U\right)=\lim _{\xi \rightarrow \infty} H=0$. Значит, максимум $w$ в области $D_{\xi}$ при любом $\xi$ неположителен, следовательно, $w \leqslant 0$ в области $D_{\infty}=\left\{(x, t): t \geqslant 0, x \in\left[0, S_{0}\right]\right\}$. Теорема 3 доказана.

Итак, воспользуемся полученной оценкой, чтобы оценить разность $h(t)-S_{0}$.

Теорема 4. Имеет место оценка $0 \leqslant S_{0}-h(t) \leqslant \sqrt{D /(2 c)} e^{q t / 2}$.

Д о к а з а т е л ь с т в о. Первое неравенство очевидным образом следует из того, что

$$
h(t)=U(t, h(t))<V_{\infty}(h(t)) .
$$


Для доказательства второго неравенства запишем:

$$
\begin{aligned}
D e^{q t} & \geqslant D e^{p S+q t} \cos (\lambda S) \geqslant V_{\infty}(h(t))-U(t, h(t)) \\
& =V_{\infty}(h(t))-V_{\infty}\left(S_{0}\right)+\left(S_{0}-h(t)\right) \\
& =\left(V_{\infty}^{\prime}\left(S_{0}\right)-1\right)\left(h(t)-S_{0}\right)+\frac{1}{2} V_{\infty}^{\prime \prime}(\zeta)\left(h(t)-S_{0}\right)^{2}
\end{aligned}
$$

где $h(t) \leqslant \zeta \leqslant S_{0}$.

Вторая производная $V_{\infty}^{\prime \prime}(\zeta)$ не меньше, чем $V_{\infty}^{\prime \prime}\left(S_{0}\right)=2 c$. Таким образом, имеем $S_{0}-h(t) \leqslant \sqrt{D /(2 c)} e^{q t / 2}$.

\section{СПИСОК ЛИТЕРАТУРЫ}

1. Shepp L., Shiryaev A. N. The Russian option: reduced regret. - Ann. Appl. Probab., 1993, v. 3, № 3, p. 631-640.

2. Graversen S. E., Shiryaev A.N. An extension of P. Lévy's distributional properties to the case of Brownian motion with drift. - Bernoulli, 2000, v. 6, № 4, p. 615-620.

3. Peskir G., Shiryaev A.N. Optimal Stopping and Free-Boundary Problems. Basel: Birkhäuser, 2006, $500 \mathrm{p}$.

4. Ekström E. Russian options with a finite time horizon. - J. Appl. Probab., 2004, v. 41, № 2, p. 313-326.

5. Bian B.J., Dai X.L., Yuan G.Q. Asymptotic analysis and numerical computation of American option when expiry date runs to infinity. (Chinese). - Tongji Daxue Xuebao Ziran Kexue Ban (J. Tongji Univ.), 2005, v. 33, № 4, p. 545-549.

Поступила в редакцию 12.XII.2005

Исправленный вариант 25.III.2008 